\title{
Inclusão digital e uso de tecnologias dinformação: a saúde do adolescente em foco
}

Ricardo Bezerra Cavalcante

Pós-doutorado em Ciência da Informação ECI/UFMG. Doutorado em Ciência da Informação - ECI/UFMG

Juliana de Jesus Silva

Enfermeira, Mestre em Saúde Pública - Faculdade de Medicina da Universidade Federal de Minas Gerais

Jessica Rauane Teixeira Martins

Enfermeira, Mestranda do Programa de Pósgraduação em Enfermagem da Universidade Federal de São João Del Rei

Tamires Rezende Passos

Enfermeira, Membro do Núcleo de Estudos e Pesquisas em Avaliação e Gestão de Serviços de Saúde da Universidade Federal de São João Del Rei

Talita Ingrid Magalhães

Enfermeira, Mestrado em Enfermagem Programa de Pós-graduação em Enfermagem da Universidade Federal de São João Del Rei

Cristiano José da Silva Esteves

Enfermeiro. Especialista em Informática em Saúde. Mestrado em Enfermagem - Programa de Pós-graduação em Enfermagem da Universidade Federal de São João Del Reí

http://dx.doi.org/10.1590/1981-5344/2539

Estudo de caso único, de abordagem quanti-qualitativa que buscou analisar a inclusão digital de adolescentes em uma escola pública do Oeste de Minas Gerais. Coletaramse dados a partir de um questionário; um ambiente virtual de aprendizado e diários de campo. Os dados 
quantitativos foram analisados por estatística descritiva. Já os dados qualitativos foram analisados a partir da Análise de Conteúdo. Constataram-se fragilidades na inclusão digital dos adolescentes estudados e diferenças comportamentais no uso do ambiente virtual de aprendizado. A linguagem foi coloquial, sintética e com erros. $A$ interação foi incipiente, ocorrendo, eventualmente, o cyberbullying. O ambiente virtual foi subutilizado e constataram-se fragilidades no suporte da comunidade escolar para o acesso a tecnologia. Concluise que a inclusão digital dos adolescentes, visando o acesso à informação em saúde, no cenário estudado permanece como um desafio atual.

Palavras-chave: Inclusão digital; Acesso a informação; Informação em saúde.

\section{Digital inclusion and information technology use: the adolescent health focus}

A unique case study, of quantitative-qualitative approach, that sought to analyze the digital inclusion of adolescents in a public school in the West of Minas Gerais. Data were collected from a questionnaire; $A$ virtual learning environment and registry of observations. Quantitative data were analyzed by descriptive statistics. The qualitative data were analyzed from the Content Analysis. There were weaknesses in the digital inclusion of the adolescents studied and behavioral differences in the use of the virtual learning environment. The language was colloquial, synthetic and with errors. The interaction was incipient, eventually occurring cyberbullying. The virtual environment was underutilized and fragilities were found in the support of the school community for access to technology. It is concluded that the digital inclusion of adolescents, aiming at access to health information, in the scenario studied remains a current challenge.

Keywords: Digital inclusion; Access to information; Health information.

Recebido em 14.09.2015 Aceito em 13.07.2017 


\section{Introdução}

A inclusão digital é entendida como um processo dinâmico que permite ao indivíduo ter acesso à informação por meio de artefatos tecnológicos (BOLZAN; OLIVEIRA; LOBLER, 2013). Dentre as tecnologias disponíveis para a busca de informações, destaca-se a internet, uma vez que esta tecnologia permite obter informações de forma fácil, rápida, em qualquer local e horário (GARBIN; GUILAN; NETO, 2012). Permite também o acesso aos mais variados tipos de informação, como por exemplo, informações sobre saúde. Sendo assim, a internet representa uma importante aliada para ampliar as possibilidades de acesso a informação e conhecimentos que são a base para o "Empowerment" da população, sendo uma estratégia fundamental para a promoção da saúde (BRASIL. Ministério Público, 2002; CARVALHO, 2004).

Para Coelho, Augusto e Cardoso (2013) a internet é um forte instrumento para a prevenção de doenças e promoção da saúde. Mas é preciso considerar alguns aspectos na utilização da internet como fonte de informação sobre saúde: a qualidade da informação, a forma como são apresentadas para o público e a seriedade dos responsáveis pela informação.

Ressalta-se também que a inclusão digital não se restringe apenas à necessidade de garantir o acesso à informação por meio da internet ou outra tecnologia, o que seria uma simplificação desta problemática (GARBIN; GUILAN; NETO, 2012). É preciso desenvolver habilidades para utilizar destas tecnologias para acessar, selecionar as melhores fontes de informação e apropriar-se delas para intervir sobre a sua própria saúde, bem como transformar sua realidade (TAVARAYAMA; SILVA; MARTINS, 2012). Nesse contexto, o comportamento de uso destas tecnologias deve ser elucidado, em vários grupos sociais, a fim de compreender a interação destes indivíduos com estas tecnologias, as finalidades de sua utilização e os riscos sociais e à saúde que emergem neste contexto (CUNHA, 2008). Dentre os grupos sociais que necessitam serem incluídos digitalmente estão os indivíduos compreendidos na fase da adolescência.

A adolescência é um período de intensas transformações e nesta fase estes indivíduos apresentam-se mais vulneráveis às condições de saúde e doença, sendo importante desenvolver estratégias para promover a saúde destes indivíduos (JUNIOR et al., 2013). As tecnologias da informação e comunicação podem ser importantes aliadas neste processo, uma vez que podem tornar os conteúdos apresentados mais atrativos (DOMINSKI et al., 2013). Segundo Mano (2008), o interesse que os adolescentes possuem por tecnologias produzem auto-motivações que repercutem positivamente no aprendizado e em suas escolhas na sua trajetória de vida. Isso remete a necessidade de incorporá-las no ambiente escolar (DOMINSKI et al., 2013), no contexto familiar e comunitário (GARBIN; GUILAM; NETO, 2012) e também com vistas à promoção de sua saúde e prevenção de doenças (CHISOLM; JOHNSON; MCALEARNEY, 2011; KORCHMAROS; YBARRA; MITCHELL, 2015). Por outro 
lado, a internet e outras tecnologias podem reforçar a medicalização da sociedade e a submissão a este modelo biomédico, visto que a "ciência (bio) médica hegemônica é a principal base teórico-cognitiva das informações na rede" (GARBIN; GUILAM; NETO, 2012, p. 359).

Enfim, as tecnologias da informação estão cada vez mais presentes no cotidiano dos adolescentes, porém são necessários estudos que abordem o comportamento dos adolescentes frente a estas tecnologias, 0 que coloca esta temática dentro do contexto da "avaliação de novas tecnologias e sua aplicabilidade" definida pelo Ministério da Saúde Brasileiro como uma das prioridades de pesquisa em saúde (BRASIL. Ministério Público, 2011). Além disso, é necessário repensar os processos informacionais em saúde de modo que a inserção das tecnologias no contexto da adolescência seja reconhecida como um fator potencial de empoderamento destes sujeitos, proporcionando o desenvolvimento da autonomia em seu processo de saúde/doença/cuidado.

Diante disso, o presente estudo buscou analisar a inclusão digital de adolescentes escolares e o uso de tecnologias da informação visando o acesso à informação em saúde.

\section{Metodologia}

Trata-se de estudo de caso único, de abordagem quanti-qualitativo, realizado em uma escola da rede estadual de ensino em um município de médio porte, localizado no Oeste de Minas Gerais, sede da Superintendência Regional de Saúde, referência para 54 municípios de menor porte. A escolha desta escola foi intencional devido ao grande número de adolescentes em baixa condição sócio-econômica. Outra justificativa de escolha da escola é o fato do acesso facilitado aos pesquisadores proporcionado pela diretoria.

$\mathrm{O}$ estudo foi desenvolvido em duas fases. Na primeira fase, predominantemente quantitativa, a população do estudo foi composta por adolescentes do $6^{\circ}$ ano do ensino fundamental ao $3^{\circ}$ ano do ensino médio da referida escola, compreendendo as idades de 13 a 19 anos. Foram utilizados como critérios de inclusão a anuência dos pais através da autorização por escrito por meio do Termo de Consentimento Livre e Esclarecido, frequentar a escola no período da coleta dos dados e desejar participar da pesquisa. O cenário do estudo possuía o total de 307 alunos matriculados e destes $232(75,6 \%)$ foram incluídos. Nesta fase, para coleta dos dados foi utilizado um questionário semiestruturado baseado no modelo de avaliação denominado Inclusão Digital e Educação Informacional para a Saúde (IDEIAS), que é baseado em competências digitais, informacionais e sociais (CUEVAS; SIMEÃO, 2011). Neste artigo, serão abordadas as competências digitais avaliadas, são elas: Acesso às Tecnologias da Informação; Uso das Tecnologias da Informação; Leitura digital (formas de leitura por meio de tecnologias); Redes digitais (participação dos adolescentes em redes sociais digitais); Avaliação das Tecnologias da Informação utilizadas pelos adolescentes; Ética das 
Tecnologias da Informação (uso responsável pelos adolescentes das tecnologias da informação). Os dados coletados nesta primeira fase foram organizados e analisados por meio de estatística descritiva.

$\mathrm{Na}$ segunda fase do estudo buscou-se o aprofundamento na compreensão do uso das tecnologias da informação por estes adolescentes, mas a partir de uma abordagem qualitativa. Assim, foram incluídos adolescentes do $1^{\circ}$ ano do ensino médio da referida escola, com idade entre 14 e 16 anos. A escolha pelo $1^{\circ}$ ano foi definida por sorteio aleatório. Todos os adolescentes aceitaram participar desta segunda fase do estudo, totalizando 40 participantes. Para a coleta dos dados foi desenvolvido um Ambiente Virtual de Aprendizado (AVA) na plataforma moodle para a realização de fóruns de discussão de temáticas envolvendo a adolescência. Foram discutidas 9 temáticas, sendo elas: Adolescência e puberdade; Influência do grupo na adolescência; Relação do adolescente com os pais e familiares; Relação do adolescente com a escola; Violência; Drogas; Gravidez na adolescência; Bulimia e Anorexia na adolescência; Acne na adolescência. Cada temática foi trabalhada em um mês, totalizando 9 meses (março a novembro de 2013) de coleta.

Para a discussão das temáticas os alunos foram previamente cadastrados no AVA e capacitados para a utilização do mesmo. Os adolescentes tinham acesso ao ambiente virtual no laboratório da escola, e também podiam acessá-lo em outros locais, inclusive em casa. Havia tutores presenciais e a distância mediando às discussões. Nos encontros presenciais, no laboratório de informática da escola, os adolescentes tinham cinquenta minutos para a discussão de cada temática no fórum de discussão do AVA, e podiam realizar para a mesma temática quantas postagens achassem necessário. Em cada encontro no laboratório havia uma média de 30 adolescentes presentes, pois alguns faltavam à escola nestes dias.

No AVA havia questões problematizadoras sobre a temática abordada, e os alunos eram incentivados a participar de fóruns, leituras de textos e vídeos sobre a temática. Ainda para a coleta, foi utilizada a observação participante dos encontros presenciais no laboratório de informática, onde foi observado o comportamento dos estudantes durante a utilização dos computadores.

A observação participante é um processo onde o pesquisador se insere como observador de um determinado grupo social, entrando em contato direto com os sujeitos da pesquisa, com objetivo de coletar dados e investigar cientificamente o contexto no qual está inserido. 0 instrumento mais utilizado é o diário de campo, onde são anotadas as informações observadas (MINAYO; DESLANDES; GOMES, 2011). Essa técnica de coleta foi escolhida com o intuito de observar o comportamento e as atitudes dos adolescentes durante o período de utilização do AVA. Assim, o mesmo grupo de adolescentes foi observado durante os nove encontros presenciais no laboratório de informática da escola. As impressões que emergiam destas observações foram registradas em diário de campo. Foi utilizado um roteiro para direcionar as observações. Este 
roteiro continha aspectos a serem observados, são eles: relação do adolescente com as tecnologias; interação dos adolescentes entre si; interação dos adolescentes com os tutores; o apoio da comunidade escolar para o desenvolvimento das atividades.

O AVA, onde as temáticas foram discutidas, gera automaticamente um relatório de participação de cada adolescente. Assim, os dados provenientes deste relatório de utilização do AVA foram analisados a partir de estatística descritiva (frequência simples). Realizou-se também a análise dos registros no diário de campo. A análise desses registros foi realizada a partir da Análise de Conteúdo. Existem diferentes tipos de análise de conteúdo, que podem ser: de expressão, das relações, de avaliação, de enunciação e categorial temática (MINAYO, 2010). A autora enfatiza que por meio da Análise de Conteúdo é possível ir em busca da descoberta do que está por trás dos conteúdos evidentes, indo além do que está sendo comunicado.

A análise de conteúdo possui as seguintes etapas: Pré-análise, Exploração do material e Tratamento dos resultados/inferência/interpretação. A primeira etapa refere-se à realização de uma primeira leitura do material coletado. Nesta etapa buscou-se obter uma visão geral de todo o material e identificar as particularidades do material. Realizou-se ainda a elaboração das hipóteses base para a análise e determinamos o método de classificação do material. A segunda etapa representa a análise aprofundada do material coletado. Nesta etapa realizou-se a distribuição de partes do material coletado no método de classificação escolhido na primeira etapa, identificando os núcleos de sentido apresentados em cada classe. Além disso, foi determinado as categorias a serem discutidas: a linguagem escrita e falada pelos adolescentes durante a utilização de tecnologias; a interação entre os adolescentes e com os tutores; a utilização das ferramentas tecnológicas disponibilizadas; o suporte da comunidade escolar para o acesso a tecnologia. Na etapa final, a qual se refere ao tratamento dos dados coletados foi descrito o que foi encontrado a partir dos objetivos e pressupostos da pesquisa.

O estudo foi aprovado por Comitê de Ética em Pesquisa sob o parecer número 119.841/2012.

\section{Resultados e discussão}

Em relação ao perfil dos participantes, este estudo contou com a participação de 232 adolescentes em idade escolar, destes $115(49,6 \%)$ do gênero feminino e $107(46,1 \%)$ do gênero masculino, uma parcela de $10(4,3 \%)$ dos participantes não responderam a esta variável. Em relação a variável idade, observou-se a distribuição de 10 a 12 anos (24,1\%), 13 a 15 anos $(46,1 \%), 16$ a 19 anos $(25,1 \%)$, além de $11(4,7 \%)$ participantes que não responderam.

Constatou-se que $70 \%$ dos participantes possuíam computador em casa e com acesso a internet, sendo este o seu principal local disponível 
para acesso. A telefonia móvel emergiu como a segunda maior forma, predominante, de acessar a internet (20\%). O restante dos adolescentes $(10 \%)$ acessava a internet por outros locais (lan house, casa de colegas e vizinhos, escola). O que chamou a atenção foi o fato de que apenas $5,7 \%$ dos 232 adolescentes utilizavam a internet para buscar informações sobre saúde e doença. O que demonstra o baixo reconhecimento por parte destes adolescentes das possibilidades de se informarem, por meio da internet, sobre doenças e possibilidades de prevenção. Há na literatura o reconhecimento de que a internet pode ser utilizada por adolescentes para o suporte na gestão e prevenção de doenças (CHISOLM; JOHNSON; MCALEARNEY, 2011) assim como na orientação da iniciação sexual (KORCHMAROS; YBARRA; MITCHELL, 2015). Entretanto, destaca-se que estas informações relacionadas à saúde ou a doença são buscadas pelos adolescentes quando estão envolvidos em situações que tornam estas informações significativas, como o desejo de entender o processo da doença quando já são acometidos por ela, ou diante de um familiar ou amigo enfermo (CHISOLM; JOHNSON; MCALEARNEY, 2011). Também quando desejam iniciar a sua atividade sexual buscam informações por meio da internet, devido a privacidade relacionada a este assunto (KORCHMAROS; YBARRA; MITCHELL, 2015). Assim, o desafio que emerge é de tornar as informações sobre saúde e doença significativas para os adolescentes não somente quando já são acometidos por um processo patológico, mas a busca da informação na internet para a prevenção de doenças é um comportamento que precisa ser incentivado. Além disso, qualificar as informações de sites que se destinam a disseminar informações sobre saúde e doença é outra necessidade (MORETTI; OLIVEIRA; SILVA,2012).

Em relação à avaliação da competência digital "Acesso às TICs" verificou-se que entre as aplicações informáticas utilizadas pelos adolescentes obtivemos 343 repostas, distribuídas da seguinte forma: processadores de texto (37,9\%), jogos eletrônicos $(29,45 \%)$, programas de criação de apresentações $(15,74 \%)$, folhas de cálculo $(8,45 \%)$, banco de dados $(4,96 \%)$ e outros $(3,50 \%)$. Sobre a utilização de aplicações para a internet foram 537 respostas, destas o uso de buscadores foi predominante $(34,64 \%)$, seguidos pelos correios eletrônicos $(22,16 \%)$, navegadores $(17,69 \%)$, mensagens instantâneas $(13,41 \%)$, chats $(10,43 \%)$ e fóruns $(1,68 \%)$.

Outra competência avaliada foi o "Uso das TIC's". O uso de canais de alerta de notícias, RSS ou Feeds de sites especializados em saúde parece pouco difundida entre os adolescentes, visto que $70 \%$ dos 232 adolescentes afirmaram não usar desta ferramenta. Novamente, isto reforça o apontamento anterior de que a maioria dos adolescentes estudados não atribuem significado às informações sobre saúde/doença/cuidado no contexto da internet.

Sobre o uso de ambientes virtuais de aprendizagem, cerca de $83 \%$ dos adolescentes nunca tinham utilizado. Este achado aponta para a necessidade de maior visibilidade da Educação a Distância no contexto da 
educação básica. As tecnologias que envolvem a educação à distância podem ser utilizadas por estes adolescentes em diversos contextos: desenvolvimento de disciplinas; projetos promovidos na própria escola; reforço escolar e até mesmo a preparação destes adolescentes para o mercado de trabalho ou para outros contextos de continuidade de seus estudos (MONTEIRO, 2014). A metodologia blended learning (atividades presenciais e a distância) pode ser utilizada como uma estratégia de operacionalização deste processo (SILVA, 2014). Entretanto, é preciso que a própria comunidade escolar seja conscientizada da importância da educação à distância no cenário da escola, como uma possibilidade para os educandos e para os professores. Além disso, a educação a distância deve ser inserida em uma política ampla de incorporação das tecnologias da informação no contexto da educação básica (MONTEIRO, 2014).

Sobre a competência de realizar "Leitura digital", a maioria dos 232 adolescentes declarou realizar a leitura diretamente na tela do computador $(81 \%)$, seguidos dos que lêem na tela e posteriormente imprimem $(16,74 \%)$ e os que imprimem para posterior leitura $(2,26 \%)$. Já o uso de suporte para a leitura de arquivos, foram fornecidas 385 respostas em que o uso de porta USB e Pen Drive $(41,82 \%)$, o telefone celular $(27,27 \%)$, CD ou DVD $(15,58 \%)$, Tablets $(8,57 \%)$ e e-book $(6,75 \%)$ foram relacionados.

A competência de participação em "Redes sociais digitais" também foi avaliada. Dos 232 adolescentes questionados, afirmaram participar $(60,09 \%)$, seguidos pelos que não participam $(33,48 \%)$ e pelos que não sabem responder $(6,44 \%)$. E quando o assunto é a participação nas redes sociais, em comunidades relacionadas à saúde, a maioria não participa $(92,65 \%)$. Neste contexto, as redes sociais digitais que poderiam ser utilizadas por estes adolescentes visando a troca de informações sobre prevenção de doenças e cuidados também não são utilizadas. Emerge o desafio de incluir estes adolescentes no contexto das redes sociais digitais, mas envolvendo também o compartilhamento de informações e conhecimentos sobre saúde/doença/cuidado. E principalmente informações qualificadas, de fontes seguras, que potencialmente contribuam para a promoção da saúde destes indivíduos. As redes sociais têm se tornado uma das principais tecnologias para os adolescentes se inserirem socialmente (LIMA et al., 2012). Permitem ao adolescente uma interação fácil, rápida e em larga escala. Um estudo que avaliou a utilização do computador por adolescentes identificou que $85 \%$ destes adolescentes utilizam o computador para acessar as redes sociais (SPIZZIRRI et al., 2012). Tal fato demonstra a afinidade que os adolescentes possuem por redes sociais enquanto instrumento de busca e disseminação de informações, podendo estas serem articuladas às necessidades de educação, mas também no contexto de sua própria saúde e na prevenção de doenças. Neste sentido, as redes sociais digitais são mais atrativas, permitem a exposição de duvidas e opiniões sobre o conteúdo, promovendo uma atitude ativa no processo de aprendizagem (FERREIRA; MOTA, 2014). 
Em relação à competência "Avaliação das TIC's", com relação a facilidade de acesso e conectividade dos 232 participantes, observou-se que a maioria $(63,98 \%)$ conecta-se a internet com facilidade e rapidez, seguidos pelos que tem facilidade mas nem sempre a conexão funciona $(27,12 \%)$ e dos que não conectam-se facilmente a internet $(8,90 \%)$. Enfatizamos, com estes resultados, que a conectividade ainda precisa ser ampliada com vistas à inclusão digital destes adolescentes. Este desafio é uma responsabilidade governamental, política e também da própria comunidade escolar. Ampliar as possibilidades de acesso às tecnologias da informação, à internet e, por conseguinte a conectividade não deve ser apenas um interesse pessoal, uma responsabilidade do cidadão, mas uma política de estado, um conjunto de estratégias que envolvam as instâncias públicas responsáveis pela garantia do direito constitucional de acesso a informação (BERCHARA, 2008). Ressalta-se que atualmente o acesso à informação é reconhecido como uma variável de grande valor para a transformação da realidade social, principalmente pelo fato de que a participação social depende da formação de um cidadão crítico, reflexivo e capaz de intervir em sua realidade (SOUSA; MIOTA; CARVALHO, 2011).

A "Ética das TICs" foi outra competência investigada, onde se avaliou o reconhecimento por parte dos adolescentes da importância do uso responsável das TICs. Assim, dos 232 respondentes a maioria $(54,94 \%)$ relatou ter muita importância utilizar as tecnologias de forma responsável. Porém há adolescentes que entendem o uso responsável das TICs como algo de pouca importância $(41,2 \%)$, bem como há outros que afirmaram que esta temática não possui nenhuma relevância $(3,86 \%)$. Isto é preocupante, pois se sabe que os adolescentes estão em uma fase da vida relacionada às descobertas, aos desafios, à exposição aos riscos e à necessidade de compreensão dos limites e das regras que se estabelecem no convívio social (BRASIL. Ministério Público, 2005; SILVA, 2014). Em tempos de crimes cibernéticos, exibicionismos e outros perigos digitais (MALAR, 2012), a necessidade do uso das tecnologias da informação de forma responsável e comprometido com a ética social é um desafio a ser suplantado no contexto da adolescência. Este é um aspecto que poderia ser desenvolvido juntamente com estes indivíduos no seio familiar, mas também no contexto da escola. A construção que se faz necessária é a compreensão, por parte destes indivíduos, que as tecnologias da informação podem ampliar possibilidades, mas também podem trazer prejuízos para si e para o outro, e isto depende da sua forma de utilização.

Ainda, nesta mesma competência, verificou-se que a maioria dos adolescentes $(62,34 \%)$ compartilham com outros as suas habilidades tecnológicas, seguidos pelos que não compartilham $(37,66 \%)$. Assim, além dos aspectos éticos que precisam ser difundidos entre os adolescentes ao se utilizar de tecnologias da informação, é necessário também promover entre os mesmos o comportamento de "compartilhar" informações e tecnologias. $O$ aprendizado colaborativo precisa ser difundido no ambiente escolar, no contexto do processo ensino- 
aprendizagem, na comunidade e em outros cenários de inserção do adolescente. Principalmente, nesta fase, na adolescência, onde a convivência em grupos é um comportamento característico destes indivíduos (WAASDORP, 2013). O aprendizado colaborativo é um método no qual é necessário a interação entre todos os envolvidos para que construam o conhecimento sobre determinado tema. Todos os envolvidos trabalham em equipe se relacionando e se complementando diante do objetivo proposto (DIAS; FRANÇA; SILVA, 2013). Assim, promover a socialização e compartilhar saberes/habilidades são fundamentais para a disseminação de conhecimentos sobre saúde que potencialmente transformam a realidade social.

Analisando o relatório de utilização do AVA, entre as temáticas discutidas a que apresentou maior número de postagens dos adolescentes no AVA foi a temática Relação familiar (32\%) seguida por Relação do adolescente com a escola (14\%) e Influência do grupo na adolescência $(13 \%)$, como mostrado na Tabela 1.

Tabela 1 - Postagens de adolescentes por temática, no AVA

\begin{tabular}{lcc} 
Temática & $\mathbf{n}^{\star}$ & $\%$ \\
\hline \hline Relação familiar & 63 & 32 \\
Relação dos adolescentes com a escola & 27 & 14 \\
Influência do grupo na adolescência & 26 & 13 \\
Drogas & 24 & 12 \\
Violência & 24 & 12 \\
Bulimia e anorexia & 18 & 9 \\
Puberdade & 5 & 3 \\
Acne & 5 & 3 \\
Gravidez na adolescência & 5 & 3 \\
\hline Total & $\mathbf{1 9 7}$ & $\mathbf{1 0 0}$ \\
\hline Fonte: Elaborada pelos autores (2015). & & \\
$\quad *$ Número de postagens realizadas no AVA pelos adolescentes.
\end{tabular}

Quando analisadas as postagens em relação ao sexo, verificou-se que dentre as temáticas abordadas, os adolescentes do sexo feminino apresentaram maior número de postagens na temática Relação familiar (29\%), tendo ainda a distribuição de respostas mais homogêneas entre as demais temáticas. Em contrapartida, os adolescentes do sexo masculino concentraram as suas respostas nas temáticas Relação familiar $(36 \%)$ e influência do grupo na adolescência (16\%) (Tabela 2). Ainda desperta atenção o fato de que as temáticas puberdade, acne e gravidez na adolescência apresentaram, conforme tabela 2, o menor percentual de postagens em ambos os sexos. 
Tabela 2 - Postagens de adolescentes por sexo e por temática, no AVA

\begin{tabular}{lcccc} 
Temática & \multicolumn{2}{c}{ Feminino } & \multicolumn{2}{c}{ Masculino } \\
& $\mathbf{n}^{\star}$ & \% & $\mathbf{n}^{*}$ & \% \\
\hline \hline Puberdade & 2 & 2 & 3 & 4 \\
Acne & 2 & 2 & 3 & 4 \\
Gravidez na adolescência & 2 & 2 & 3 & 4 \\
Influência do grupo na adolescência & 13 & 11 & 13 & 16 \\
Relação Familiar & 34 & 29 & 29 & 36 \\
Relação dos adolescentes com a escola & 18 & 15 & 9 & 11 \\
Drogas & 16 & 14 & 8 & 10 \\
Bulimia e anorexia & 12 & 10 & 6 & 7,5 \\
Violência & 18 & 15 & 6 & 7,5 \\
\hline Total & $\mathbf{1 1 7}$ & $\mathbf{1 0 0}$ & $\mathbf{8 0}$ & $\mathbf{1 0 0}$ \\
\hline
\end{tabular}

Fonte: Elaborada pelos autores (2015).

*Número de postagens realizadas no AVA pelos adolescentes.

O interesse de adolescentes, de ambos os sexos, por discussões realizadas em fóruns de AVAs se diferenciam em algumas temáticas (GOMES; SCHERER; LÔBLER, 2012) conforme verificado neste estudo. Entretanto, ainda há a possibilidade de desinteresse para a discussão de algumas temáticas e o entendimento de que são exclusivas para um gênero em detrimento do outro, como é o caso da gravidez na adolescência. A falta de significado de algumas temáticas, como a acne, também podem representar um obstáculo para a ampliação do compartilhamento de informações. Assim, é necessário o envolvimento destes adolescentes de ambos os gêneros com estas temáticas a fim de que acessem este tipo de informação, compreendam suas evoluções e saibam como se autocuidar.

Ao analisar a linguagem escrita e falada pelos adolescentes durante a utilização do ambiente virtual, constatou-se que a mesma foi coloquial, sintética, com presença de gírias, erros ortográficos e de concordância. As pontuações que organizam a estrutura frasal também não foram utilizadas. Parece não haver uma preocupação com a estética da escrita: "Eu axo q a violencia uma coisa mto ruim todos teriam q pensar um pouco se deveria realmente praticar pois afinal ela so tras 0 mau tanto para quem sofre quanto pra quem pratica $[\ldots]^{\prime \prime}$ (Adol. 23; Temática violência).

Por outro lado há ênfase na velocidade da comunicação gerando reducionismos nas palavras e nas frases: "eu percebe que o nosso corpo motifica muito tem coisas que muda que vc nao percebe tipo a voz, o tamanho, a maturiida..." (Adol. 16; Temática puberdade)

Nos encontros presenciais, no laboratório de informática da escola, os adolescentes apresentaram uma linguagem muito próxima da 
linguagem utilizada nos fóruns do ambiente virtual, como foi observado e registrado em diário de campo: Os adolescentes, mesmo diante dos computadores, conversam entre si e utilizam uma linguagem característica da idade, repleta de gírias, jargões [...]. (Registro 4; Diário de campo). Outro trecho também destaca: A conversa paralela entre os adolescentes acontecem a todo momento. Eles falam de um jeito que as vezes não é possível entender. (Registro 5; Diário de campo).

A aproximação da escrita com o discurso oral faz com que os internautas tanto no meio virtual quanto na realidade utilizem diálogos parecidos para se comunicar (SOUZA; DEPS, 2012). Os adolescentes buscam expressar suas emoções por meio da junção dos diálogos do diaa-dia e a escrita no meio virtual, o que facilita a comunicação e o entendimento, já que as expressões utilizadas são comuns entre eles (GOMES; CORREA, 2009). Além disso, a internet exige maior velocidade para a leitura e comunicação entre os internautas, o que faz com que adolescentes criem formas mais rápidas de se comunicar por meio da escrita. Ao fugir das regras gramaticais impostas pela norma culta, os adolescentes acabam criando as suas próprias regras de linguagem no meio virtual, que podem ser mais rígidas do que as impostas pela língua padrão (SOUZA; DEPS, 2012).

A escrita fora do convencional no meio virtual tem se tornado um modismo, o que caracteriza essa forma de comunicação entre os adolescentes como um modelo estabelecido e de mútuo entendimento. A escrita padrão dificulta a interação em tempo real entre os adolescentes, 0 que faz com que eles adotem a forma rápida e muitas vezes errônea de escrever para acelerar a comunicação (GOMES; CORREA, 2009). Entretanto, as regras sociais vão além do mundo digital. O adolescente precisa se submeter às regras sociais que também estão presentes virtualmente, dentre elas, a comunicação por meio de uma linguagem mais elabora, inteligível e aceita socialmente.

Verificou-se que nos fóruns de discussão a interação entre os adolescentes e com os tutores, com vistas ao desenvolvimento do tema proposto, foi incipiente. Restringiram-se em responder às questões problematizadoras propostas pelos tutores, como destacado: Questão problematizadora: "O tema é Bulimia e anorexia. Relate alguma história ou vivência sobre estes temas". (Tutor 1; Temática bulimia e anorexia). Em seguida o adolescente respondeu: "eu nunca vivenciei essa doença mas ja vi algumas reportagens relatando a doença e mostrando algumas pessoas. Essa doença acaba com a vida de qualquer pessoa. Esse e meu ponto de vista". (Adol. 8; Temática bulimia e anorexia).

Os adolescentes também não interagiam muito com os tutores, apesar de frequentemente os tutores realizarem postagens motivando a participação dos adolescentes: "[...] Participem também, estamos sentindo falta da participação de vocês!!!". (Tutor 1; Temática drogas). Outro tutor registrou: "Os adolescentes durante os encontros presenciais têm uma interação conosco mais direcionada para o esclarecimento de dúvidas sobre como utilizar o AVA". (Registro 9; Diário de campo) 
O acompanhamento do tutor na $\mathrm{EaD}$ é fundamental no aproveitamento e realização das atividades propostas. Sendo assim, o tutor se torna mais que um apoio no processo de aprendizagem, ele promove a aprendizagem através de um processo dinâmico e ativo (DEMARCO et al., 2012). O tutor, na EaD, tem o papel de mediar, facilitar e orientar o processo de ensino-aprendizagem. Além disso, deve estimular a participação dos alunos na aprendizagem colaborativa. Por se tratar de um público adolescente, é necessário também que o tutor atue adequando-se a este público. É preciso que o tutor compreenda e seja capaz de interagir em um contexto direcionado ao pensar e agir do adolescente, é necessário que a tutoria seja exercida de forma mais ativa e mais dinâmica para estimular a participação.

Outro aspecto que emergiu das análises, relacionado a interação, foi o fato de que os adolescentes praticavam no AVA, eventualmente, o cyberbullying e nos encontros presenciais o bullying. Um dos adolescentes postou o comentário: "Prazer meu nome e A. tenho 16 anos e acho que o projeto vai ser bom para todos na turma!! Minhas espectativas são boas" [escreveu de cor rosa]. (Adol. 5; Apresentação). Logo em seguida outro adolescente respondeu ao comentário: "hsuahauasus escrever igual homem junstin". (Adol. 2; Apresentação). Também um dos tutores deixou registrado em diário de campo uma ofensa a outro adolescente quando da falta de habilidade para utilizar o ambiente virtual: "Os adolescentes do sexo masculino proferem palavras ofensivas às adolescentes, como: burra, ultrapassada e idiota". (Registro 6; Diário de campo)

O cyberbullying é uma forma de preconceito expressa em ambiente virtual, e é derivado do bullying. Tem consequências psicossocioemocionais que podem ser ainda mais devastadores que 0 bullying, pois ocorre exposição de forma negativa da vida de outra pessoa por meio da tecnologia que pode ser armazenada e exposta por tempo ilimitado, além disso, há a dificuldade de descobrir o agressor e impedir estas ações (PERFEITO, 2012).

Se o AVA é um meio interessante para promover a educação e disseminar informações, por outro lado ele pode reproduzir os mesmos problemas que ocorrem no ambiente presencial, como o bullying (CANTONE et al., 2015). É importante entender qual a percepção dos adolescentes acerca das suas atitudes no meio virtual e as suas consequências, que são muitas vezes mais graves devido a facilidade de disseminação de informações amparadas por este suporte tecnológico.

O cyberbullying surge como uma forma de preconceito e intolerância àquilo que é considerado fora dos padrões da sociedade (SOUZA; SIMÃO; CAETANO, 2014). Traz consigo consequências como aumento do nível de ansiedade, uso e abuso de substâncias psicotrópicas, depressão, ideação suicida e prejuízos escolares (WENTD; LISBOA, 2014). Sendo assim, as estratégias de enfrentamento são fundamentais, visando minimizar os danos deste fenômeno, sendo a família e a comunidade escolar os agentes ativos seja no enfrentamento preventivo quanto no enfrentamento após o 
acontecimento do cyberbullying (WENTD; LISBOA, 2014; CANTONE et al., 2015).

Em relação à utilização da tecnologia, neste caso o Ambiente Virtual, constatou-se que os adolescentes não utilizaram o AVA em toda sua potencialidade: "Os adolescentes se restringem a responder as discussões nos fóruns, deixando de acessar os demais recursos disponíveis no ambiente virtual, como a biblioteca virtual". (Registro 7; Diário de campo).

Interessavam em utilizar a internet para "acessam outros sites, como o facebook, twitter e joguinhos. Parecem querer explorar outras coisas na internet". (Registro 8; Diário de campo). Estavam em busca de informações diferentes daquelas propostas e debatidas no momento do encontro presencial.

Os AVAs devem ser desenvolvidos de forma a possuir ferramentas que despertem o interesse e o desejo de sua utilização. Devem ainda ser moldados com ferramentas mais interativas principalmente pensando no público adolescente. Um AVA deve ser desenvolvido de forma a fornecer variados instrumentos para o processo de ensino-aprendizagem. Deve ser considerado neste processo de construção fatores como o conteúdo a ser ensinado e a experiência do aluno em utilizar tecnologias. Além disso, deve ser dinâmico para permitir adequações de acordo com as necessidades dos tutores e alunos (RODRIGUES; PERES, 2013), bem como desenvolver estratégias que incentivem o acesso deste ambiente. Os mediadores destes ambientes devem ser capacitados para promover a interação entre os participantes e incentivar a participação e utilização dos recursos disponíveis (DEMARCO et al., 2012).

No que tange ao suporte da comunidade escolar para o acesso a tecnologia, constatou-se sobre o laboratório de informática da escola:

O laboratório de informática da escola fica na maior parte do tempo fechado dificultando o acesso dos adolescentes aos computadores e internet. Alguns computadores não possuem mouse, outros não ligam, outros não tem teclado. (Registro 1; Diário de campo).

Outro tutor endossa esta situação: "Alguns adolescentes parecem estar entrando no laboratório de informática da escola pela primeira vez. E mostram-se desejosos em ligar os computadores, apesar de muitos não saberem como ligá-los". (Registro 3; Diário de campo).

Em relação ao apoio de professores e diretoria para o acesso à tecnologia, registrou-se: "apenas uma professora, a de biologia, participa com os adolescentes das atividades no laboratório, os demais professores só liberam os alunos para participarem" (Registro 15; Diário de campo). Outro tutor registra o envolvimento da diretoria: "a diretora hoje veio observar como as atividades acontecem no laboratório, e também 
Inclusão digital e uso de tecnologias da informação: a saúde do adolescente em foco
Ricardo Bezerra Cavalcante; Juliana de Jesus Silva; Jessica Rauane Teixeira Martins; Talita Ingrid Magalhães Silva; Tamires Rezende Passos; Cristiano José da Silva Esteves

conversou com alguns professores para participarem". (Registro 22; Diário de campo)

Estas constatações remetem à necessidade de ir além da presença de laboratório de informática na escola com computadores disponíveis. A universalização do acesso às TICs depende de equipamentos, seu adequado funcionamento, disponibilidade de acesso e suporte para a sua utilização, mas também é preciso tornar o adolescente capaz de buscar, filtrar, analisar e usar a informação para a transformação de sua própria realidade (LIMA, 2012).

Neste contexto, a escola pode contribuir disponibilizando o acesso às TICs, bem como auxiliando na construção de competências relacionados à busca, apreensão e uso da informação em sua práxis social. Em suma, o uso das TICs, na escola, além de uma política institucional necessita estar inserido na prática educacional, na administração escolar e incentivado no contexto familiar onde o adolescente está inserido (GARBIN; GUILAM; NETO, 2012). O uso das TICs na escola, além de sua ênfase de necessidade para o mercado de trabalho, precisa ser compreendido e incentivado como uma necessidade para todos os contextos da vida do adolescente, inclusive para o acesso a informações que ampliem as possibilidades de promoção de sua saúde.

\section{Considerações finais}

A inclusão digital de adolescentes escolares e o seu comportamento em utilizar destas tecnologias visando à promoção de sua saúde ainda é um desafio. Para suplantá-lo é necessário alcançar alguns objetivos: a necessidade de tornar o uso destas tecnologias para acesso sobre informação em saúde mais significativas; ampliar a conectividade; envolver toda a comunidade escolar no empenho em incluir digitalmente e informacionalmente os adolescentes utilizando o espaço da escola também para esta finalidade; promover a visibilidade das ferramentas da educação à distância como uma possibilidade no contexto escolar e também na promoção da saúde; desenvolver juntamente com os adolescentes 0 uso responsável das tecnologias da informação estabelecendo padrões aceitáveis de comportamentos e inibindo os crimes cibernéticos. Ressaltamos que o alcance destes objetivos está relacionado a um envolvimento amplo entre comunidade escolar, família, políticos e gestores públicos e privado.

\section{Agradecimentos}

Agradecemos a Fundação de Amparo a Pesquisa do Estado de Minas Gerais (FAPEMIG), pelo financiamento da pesquisa. 


\section{Referências}

BERCHARA, M. Os espaços públicos de acesso à internet. In: BRASIL. Núcleo de Informação e Coordenação do Ponto BR. Pesquisa sobre o uso das tecnologias da informação e da comunicação no Brasil: TIC Domićlios e TIC Empresas 2007. 2. ed. São Paulo: Comitê Gestor da Internet no Brasil, 2008. p. 47-50.

BOLZAN, L. M.; OLIVEIRA, J. S.; LOBLER, M. L. Efeitos sociais e afetivos das políticas públicas de inclusão digital: etnografando-se uma organização não governamental. Sociais e Humanas, Santa Maria, v. 26, n. 2, p. 434-454, maio/ago. 2013.

BRASIL. Ministério da Saúde. As cartas da promoção da saúde. Brasília: Brasília, DF, 2002.2 Disponível <www.saude.gov.br/bvs/conf_tratados.html>. Acesso em: 22 jun. 2014.

BRASIL. Ministério da Saúde. Marco legal: saúde, um direito de adolescentes. Brasília, DF, 2005.

BRASIL. Ministério da Saúde. Secretaria de Ciência, Tecnologia e Insumos Estratégicos. Departamento de Ciência e Tecnologia. Agenda nacional de prioridades de pesquisa em saúde. 2. ed. Brasília, DF:Ministério da Saúde, 2011.

CANTONE, E. et al. Interventions on bullying and cyberbullying in schools: a systematic review. Clin Pract Epidemiol Ment Health; v. 11, Suppl1 M4, p. 58-76, 2015.

CARVALHO, S. R. Os múltiplos sentidos da categoria "empowerment" no projeto de promoção à saúde. Cad. Saúde Pública, Rio de Janeiro, v. 20, n. 4, p. 1088-1095, jul./ago. 2004.

CHISOLM, D. J.; JOHNSON, L. D.; MCALEARNEY, A. S. What makes teens start using and keep using health information web sites? A mixed model analysis of teens with chronic illnesses. Telemed J E Health, v. 17, n. 5, p. 324-328, Jun. 2011.

COELHO, E. Q.; AUGUSTO, A. Q.; CARDOSO, J. E. D. Informações médicas na internet afetam a relação médico-paciente? REVBIOÉT, v. 21 , n. 1, p. 142-149, 2013.

CUEVAS, A.; SIMEÃO, E. (Orgs.). Alfabetização informacional e inclusão digital: modelo de infoinclusão social. Brasília: Thesaurus, 2011.

CUNHA, M. B. Das bibliotecas convencionais às digitais: diferenças e convergências. Perspectivas em Ciências da Informação, v. 13, n. 11, p. 2-17, 2008.

DEMARCO, D. J. et al. Avaliação da tutoria no curso de especialização em Negociação Coletiva/Modalidade a Distância (MPOG-EA/UFRGS). RENOTE, v. 10, n. 3, p.1-10, 2012. 
DIAS, A.; FRANÇA, J.; SILVA, M. Tecnologia assistiva e aprendizagem colaborativa: um survey com deficientes visual em ambiente virtual de aprendizagem a partir do modelo TAM. Nuevas Ideas en Informática Educativa TISE, v. 13, n. 1, p. 240-46, 2013.

DOMINSKI, D. K. et al. Reflexões sobre a tecnologia e adolescentes: mitos e verdades. Revista de Psicologia, v. 1, n. 20, p. 22-32, 2013.

FERREIRA, H. S.; MOTA, M. M. A visão dos alunos sobre o uso do facebook como ferramenta de aprendizagem na educação física. Revista FSA, Teresina, v. 11, n. 1, p. 188-199, 2014.

GARBIN, H. B. R.; GUILAM, M. C. R. G.; NETO, A. F. P. Internet na promoção da saúde: um instrumento para o desenvolvimento de habilidades pessoais e sociais. Physis Revista de Saúde Coletiva, Rio de Janeiro, v. 22, n. 1, p. 347-363, 2012.

GOMES, A. L.; CORREA, J. Escrita teclada x escrita padrão na produção textual: a experiência de adolescentes brasileiros. Revista Portuguesa de Educação, v. 22, n. 1, p. 71-88, 2009.

GOMES, T. C.; SCHERER, L. A.; LÔBLER, M. L. Uso das redes sociais virtuais no processo de recrutamento e seleção de pessoal: uma análise na perspectiva de profissionais de Recursos humanos. In: SIMPOI, 2012, São Paulo. Anais... São Paulo: Fundação Getúlio Vargas, 2012. p. 1-17. Disponível

em: <http://www.simpoi.fgvsp.br/arquivo/2012/artigos/E2012_T00264_PCN1 2758.pdf>. Acesso em: 20 jun. 2014.

JUNIOR, A. R. F. et al. Vivência de adolescentes em atividade de promoção da saúde. Rev Bras Enferm, Brasília, v. 66, n. 4, p. 611-6144, jul./ago. 2013.

KORCHMAROS, J. D.; YBARRA, M. L.; MITCHELL, K. J. Adolescent online romantic relationship initiation: differences by sexual and gender identification. J Adolesc, v. 40, p. 54-64, Apr. 2015.

LIMA, A. L. I. TIC na educação no Brasil: o acesso vem avançando. "E a aprendizagem?" In: BRASIL. Núcleo de Informação e Coordenação do Ponto BR. Pesquisa sobre o uso das tecnologias da informação e comunicação nas escolas brasileiras: TIC Educação 2011. São Paulo: Comitê Gestor da Internet no Brasil, 2012. p. 27-33.

LIMA, N. L. et al. Os adolescentes na rede: uma reflexão sobre as comunidades virtuais. Arquivos Brasileiros de Psicologia, Rio de Janeiro, v. 64, n. 3, p. 2-18, 2012.

MALAR, M. N. Impact of cyber crimes on social networking pattern of girls. International Journal of Internet of Things, v. 1, n. 1, p. 9-15, 2012.

MANO, S. M. F. Ambiente virtual como facilitador do diálogo sobre sexualidade entre adolescentes: desenvolvimento e avaliação de um 
multimídia educativo. 2008. 170f. Tese (Doutorado em Ensino de Biociências e Saúde) - Instituto Oswaldo Cruz, Rio de Janeiro, 2008.

MINAYO, M. C. O desafio do conhecimento: pesquisa qualitativa em saúde. 11 ed. São Paulo: Hucitec, 2010.

MINAYO, M. C.; DESLANDES, S. F.; GOMES, R. Pesquisa social: teoria, método e criatividade. 30 ed. Petrópolis: Vozes, 2011.

MONTEIRO N. A. Plano Nacional de Educação 2014-2024: as perspectivas tecnológicas nas escolas. Revista Retratos da Escola, Brasília, v. 8, n. 15, p. 489-503, jul./dez. 2014.

MORETTI, F. A.; OlIVEIRA, V. E. de; SILVA, E. M. K. da. Acesso a informações de saúde na internet: uma questão de saúde pública? Revista da Associação Medica Brasileira, São Paulo, v. 58, n. 6, p. 650-658, 2012.

PERFEITO, R. S. Ambientes escolares e sociais moldados pelo Cyberbullying e suas consequências perante crianças e adolescentes. Adolesc. Saude, Rio de Janeiro, v. 9, n. 1, p. 59-63, 2012.

RODRIGUES, R. C. V.; PERES, H. H. C. Desenvolvimento de Ambiente Virtual de Aprendizagem em Enfermagem sobre ressuscitação cardiorrespiratória em neonatologia. Rev ESC Enferm, USP, v. 47, n. 1, p. 235-41, 2013.

SILVA, A. J. de C. Desenvolvimento de uma comunidade virtual para a inserção da metodologia blended learning na Educação Básica. 2014. 135f. Dissertação (Mestrado Profissional - Área de concentração em formação de professores) - Universidade Federal de Lavras, Lavras, 2014.

SOUZA, L. P.; DEPS, V. L. A linguagem utilizada nas redes sociais e a sua interferência na escrita tradicional: um estudo com adolescentes brasileiros. In: CONGRESSO INTERNACIONAL TIC E EDUCAÇÃO, 2., 2012, Lisboa. Atas... Lisboa, 2012. p.163-180.

SOUSA, R. P; MIOTA, F. M. C. S. C; CARVALHO, A. B. G. Tecnologias digitais na educação. Campina Grande: EDUEPB, 2011.

SOUZA, S. B.; SIMÃO, A. M. V.; CAETANO, A. P. Cyberbullying: percepções acerca do Fenômeno e das estratégias de enfrentamento. Psicologia Reflexão e Crítica, v. 27, n. 3, p. 582-590, 2014.

SPIZZIRRI, R. C. P. et al. Adolescência conectada: mapeando o uso da internet em jovens internautas. Psicol. Argum, v. 30, n. 69, p. 327-335, 2012.

TAVARAYAMA, R.; SILVA, R. C. M. F.; MARTINS, J. R. A sociedade da informação: possibilidades e desafios. NUCLEUS, v. 9, n. 1, p. 267-276, abr. 2012.

WAASDORP, T. E. et al. The association between forms of aggression, leadership, and social status among urban youth. J Youth Adolesc., v. 42, n. 2, p. 263-74, Feb. 2013. 
Inclusão digital e uso de tecnologias da informação: a saúde do adolescente em foco
Ricardo Bezerra Cavalcante; Juliana de Jesus Silva; Jessica Rauane Teixeira Martins; Talita Ingrid Magalhães Silva; Tamires Rezende Passos; Cristiano José da Silva Esteves

WENTD, G. W.; LISBOA, C. S. M. Compreendendo o fenômeno do cyberbullying. Temas em Psicologia, v. 22, n. 1, p. 39-54, 2014. 